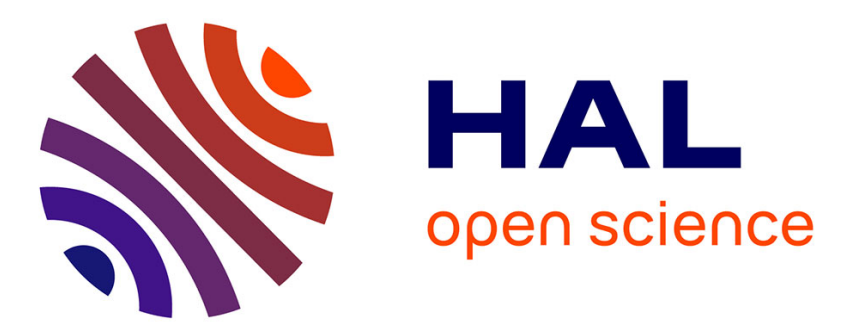

\title{
Real-time Parameters Identification of Lithium-ion Batteries Model to Improve the Hierarchical Model Predictive Control of Building MicroGrids
}

\author{
Daniela Yassuda Yamashita, Ionel Vechiu, Jean-Paul Gaubert
}

\section{To cite this version:}

Daniela Yassuda Yamashita, Ionel Vechiu, Jean-Paul Gaubert. Real-time Parameters Identification of Lithium-ion Batteries Model to Improve the Hierarchical Model Predictive Control of Building MicroGrids. 2020 22nd European Conference on Power Electronics and Applications (EPE'20 ECCE Europe), Sep 2020, Lyon, France. pp.1-10, 10.23919/EPE20ECCEEurope43536.2020.9215878 . hal03113129

\author{
HAL Id: hal-03113129 \\ https://hal.science/hal-03113129
}

Submitted on 19 Jan 2021

HAL is a multi-disciplinary open access archive for the deposit and dissemination of scientific research documents, whether they are published or not. The documents may come from teaching and research institutions in France or abroad, or from public or private research centers.
L'archive ouverte pluridisciplinaire HAL, est destinée au dépôt et à la diffusion de documents scientifiques de niveau recherche, publiés ou non, émanant des établissements d'enseignement et de recherche français ou étrangers, des laboratoires publics ou privés. 


\title{
Real-time Parameters Identification of Lithium-ion Batteries Model to Improve the Hierarchical Model Predictive Control of Building MicroGrids
}

\author{
Daniela Yassuda Yamashita, ${ }^{\mathrm{a}, \mathrm{b}}$, Ionel Vechiu ${ }^{\mathrm{a}}$ and Jean-Paul Gaubert ${ }^{\mathrm{b}}$ \\ ${ }^{a}$ ESTIA INSTITUTE OF TECHNOLOGY \\ Technopole Izarbel - 92, Allée Théodore Monod - Bidart, France \\ 'LABORATOIRE D'INFORMATIQUE ET D'AUTOMATIQUE POUR LES SYSTEMES \\ (LIAS), UNIVERSITE DE POITIERS \\ 4, Rue Pierre Brousse - Poitiers, France \\ d.yamashita@estia.fr, i.vechiu@estia.fr, jean.paul.gaubert@univ-poitiers.fr
}

\section{Acknowledgements}

The authors would like to thank the New Aquitaine Region for their financial support.

\section{Keywords}

Battery Management Systems (BMS), Control methods for electrical systems, MPC (Model-based Predictive Control), Device modelling, Microgrid

\begin{abstract}
Energy storage systems are key elements for enabling the design of MicroGrids in buildings, specially to deal with stochastic renewable energy resources and to promote peak shifting. However, inaccuracies in the batteries' mathematical models due to temperature and ageing effects can reduce the performance of a MicroGrid system. To tackle these uncertainties, this article presents a two-level hierarchical model predictive controller empowered with a data-driven algorithm for real-time model identification of Lithium-ion batteries. The objective is to enhance their state of charge estimation and to make their maximum use without damaging them. The results demonstrate that it improves up to three times the accuracy of state-of-charge estimation and increases about 3\% the annual building MicroGrid selfconsumption rate. Furthermore, the division of the building MicroGrid energy management system into two hierarchical levels soften the drawbacks arise from the inaccuracies of day-ahead data prediction while reducing the computational cost. The proposed architecture guarantees higher energetic autonomy indexes than a conventional rule-based controller in all scenarios under study.
\end{abstract}

\section{Introduction}

Building MicroGrids (BMGs) are an attractive alternative to foster the integration of renewable energy sources (RESs) into the electrical grid [1]. However, their wide implementation is restrained by the difficulty of designing a generic Energy Management System (EMS) capable of operating under stochastic variations in the power plant. Unpredictability in power generation and power consumption, along with inaccuracy of mathematical models of BMG components lead to the under exploitation of BMG resources or equipment damaging [2].

In the literature, there are various strategies to implement a reliable and efficient EMS [3]. Among the existent strategies, the hierarchical control structure enables to embed algorithms with different complexities at the same time, thanks to the parallel coordination of multiple control layers deployed at different sample time [4]. Concerning the EMS algorithms, those based on model predictive control (MPC) have proved their robustness against environmental disturbances, even with simplified plant model [5]. Nonetheless, there is a lack in evaluating the MPC performance under environmental changes, such as temperature, electric devices ageing and batteries model parameters inaccuracy. 
In order to face these uncertainties, there are several techniques to estimate better the intrinsic parameters of batteries, such as the Arrhenius equation [6], or models devised from technical specifications [2]. However, they require beforehand model calibration, which can lead to uncertainties throughout the batteries' life. In this context, strategies based on data analysis are increasingly implemented. The most pertinent algorithms are incremental analysis of the voltage and capacity to estimate the batteries' state of health [7], Kalman filter estimator [8] and other machine learning methods [9].

Therefore, this article proposes a two-level Hierarchical MPC (HMPC) with a Real-Time Model Identification (RTMI) module to deal with batteries' parameter inaccuracy. Equipped with photovoltaic (PV) panels and Li-ion batteries, the purpose of the proposed HMPC is to maximise the MicroGrid selfconsumption rate $\left(\tau_{a}\right)$ [10]. Relying on continuous data measurement and forecast data, the strategy is to optimise its internal power flow by promoting the use of renewables and reducing the energy dependency on the external grid. The results show that the RTMI enhances the State-of-Charge (SoC) estimation, leveraging $\tau_{a}$ and leading HMPC to outperform the traditional rule-based strategy.

The remaining of this paper is organised as follows. In the second section, the overview of the underlying hierarchical control structure associated with the proposed algorithm for identifying the battery parameters is described. The third section details the new model developed for the estimation of the SoC and the RTMI algorithm. The fourth section presents the performance of the proposed control strategy, by comparing the HMPC with RTMI module with both the conventional MPC under hierarchical and non-hierarchical architecture and a traditional rule-based controller. In the last section, the conclusions on the advantages and disadvantages of the proposed approach are summarized.

\section{Overview of the hierarchical energy management system}

The designed hierarchical EMS is a centralised controller for optimising the power flow of a gridconnected BMG that interacts with the external grid throughout a community aggregator, as illustrated in Fig. 1. Composed mainly by three control units, the objective of the proposed control architecture is to assure the building internal power balance with minimum dependency on the main grid. The following two subsections describe the hierarchical MPC and the RTMI module, respectively.

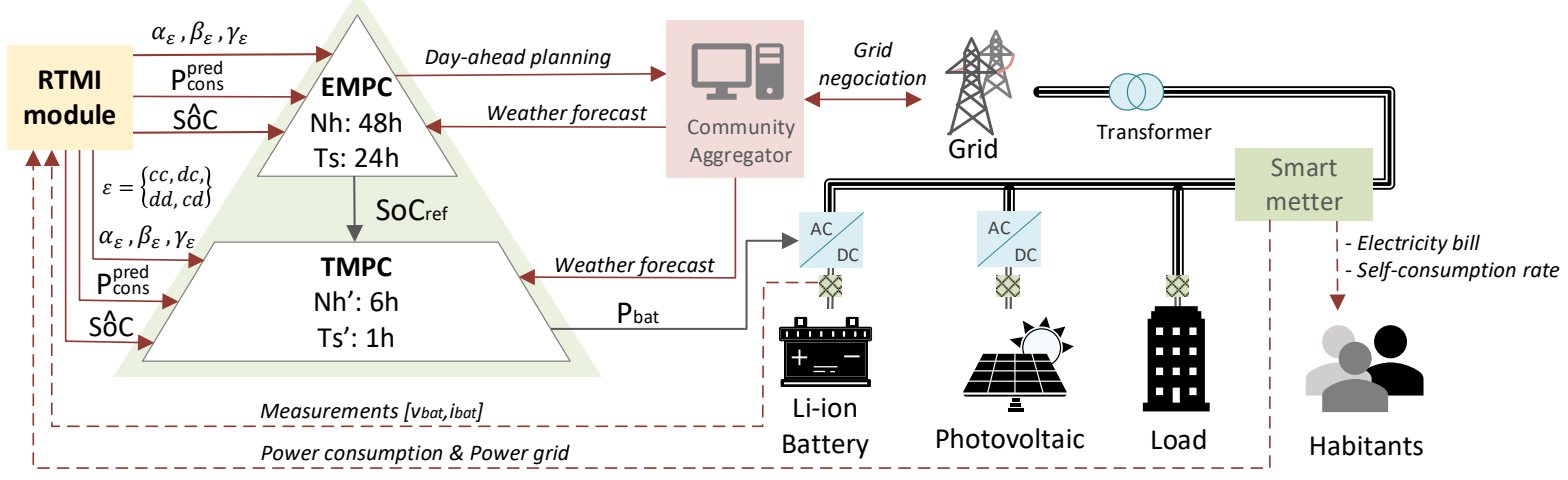

Fig. 1: Two-level hierarchical model predictive control architecture empowered with the batteries' realtime model identification module for energy management of a grid-connected building MicroGrid.

\section{Hierarchical model predictive controller}

The proposed HMPC disposes of two control levels, namely Economic MPC (EMPC) and Tracking MPC (TMPC). The upper control level performs the economic power dispatch through an EMPC and determines both the day-ahead electricity trading planning to be sent toward the community aggregator and the batteries' State-of-Charge references $\left(S o C_{r e f}\right)$ to be forward to the TMPC. Simultaneously, the lower level determines the batteries' power references $\left(P_{b a t}\right)$ based on the updated prediction data and measurements, by performing a TMPC to follow $S o C_{r e f}$.

Considering the fluctuations of the data prediction of both PV power generation and building power consumption $\left(P_{\text {cons }}^{\text {pred }}\right)$, the EMPC determines $S o C_{r e f}$ that, within a horizon of $N_{h}^{E M P C}=48 h$, minimises 
the external grid energy dependency. Therefore, the EMPC maximises $\tau_{a}$ defined by (1) and minimises additional energy imports to reduce the cost of purchasing electricity. For this, the cost function defined by $(2)$ is optimised daily $\left(T_{S}^{E M P C}=24 h\right)$, in which the total power injected $\left(P_{\text {injected }}\right)$ and the total power purchased ( $\left.P_{\text {purchased }}\right)$ are minimised. It is noteworthy that the horizon $\left(N_{h}^{E M P C}\right)$ twice bigger than the updating time $\left(T_{S}^{E M P C}\right)$ is to guarantee the most suitable $S o C$ at the end of the day. Doubling the horizon avoids unnecessary completely discharge of batteries at the end of the day since the last half of control variables determined by the EMPC is not implemented.

$$
\begin{gathered}
\tau_{a}=\frac{\text { Total energy produced from } R E S \text { that is consummed locally }}{\text { Total energy produced from RES locally }}=1-\frac{\sum_{k} P_{\text {injected }, k}}{\sum_{k} P_{P V, k}} \\
\text { So } C_{\text {ref }}=\arg \left(\min _{\text {SoC }_{r e f}} \sum_{k=1}^{N_{h}^{E M P C}=48} P_{\text {injected }, k}+P_{\text {purchased }, k}\right)
\end{gathered}
$$

Parallelly to the EMPC, the TMPC is triggered at each $T_{S}^{T M P C}=1 \mathrm{~h}$ to follow the $S o C_{r e f}$ as much as possible, considering the updated data prediction and the $\widehat{S O C}$ estimated from the last measurements. To achieve this, the TMPC minimises hourly the cost function defined by (3). The purpose of the TMPC is to reduce the stochastic variation of the day-ahead power imbalance and battery model inaccuracies, by using a shorten horizon, simplifying the optimisation process.

$$
P_{\text {bat }}=\arg \left(\min _{P_{\text {bat }}} \sum_{k=1}^{N_{h}^{T M P C}=6}\left(\text { SoC }_{r e f, k}-\widehat{\operatorname{SoC}}_{k}\right)^{2}\right)
$$

Meanwhile, in the background, the EMPC supervises the performance of the TMPC. Every hour, the EMPC compares the accuracy of day-ahead power planning sent to the aggregator $\left(P_{\text {grid }}^{r e f}\right)$ and $S o C_{r e f}$ with real measurements. As soon as the absolute difference between them is higher than a predefined threshold - named $\Delta_{S o C}^{t h r}$ or $\Delta_{P \text { grid }}^{t h r}$ at time $t_{\text {reopt }}$ - the EMPC determines new $S o C_{r e f}$ using the updated prediction data, but with a reduced horizon, as illustrated in Fig. 2. The reduced horizon comprehends the data prediction of the remaining period of the first EMPC optimisation and is equal to $N_{h}-t_{\text {reopt }}$.

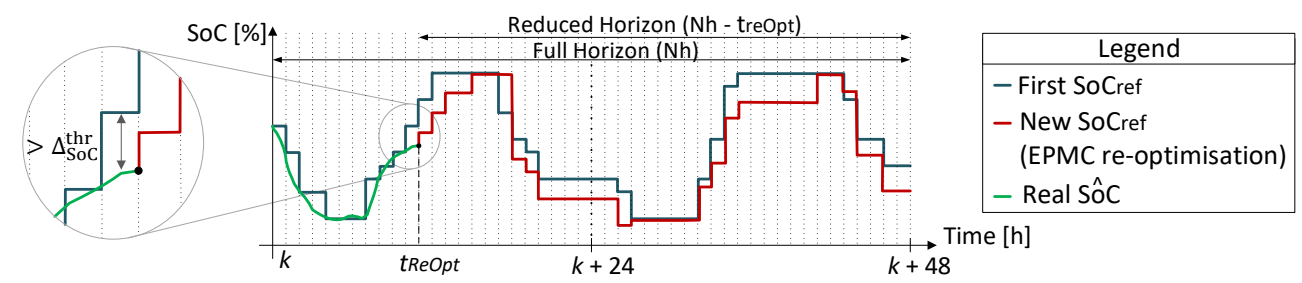

Fig. 2: Re-optimization process of EMPC with reduced horizon.

The main constraints embedded in both EMPC and TMPC are those to respect the recommendations of the French Energy Regulation Commission (ERC) for grid-connected BMGs with PV capacity over 100 $\mathrm{kWc}$ [11]. To prevent energy speculation with the local energy storage systems (ESSs) when trading electricity, the ERC allows charging the ESSs only from renewable power generated locally. Additionally, it imposes that $\tau_{a} \geq 50 \%$ at the end of the year [12]. As a result, batteries can be charged $\left(P_{b a t}^{c h}\right)$ as soon as there is an internal energy surplus, while they can be discharged $\left(P_{b a t}^{\text {dis }}\right)$ when there is an energy deficit, as detailed in (4). Moreover, aiming to extend the life of batteries, the maximum power rate $\left(P_{b a t}^{\max }\right)$ and the SoC limits defined by their manufacturer must be supervised [2], [13]. Consequently, it is important to well estimate future $\mathrm{SoC}\left(S o C_{k+1}\right)$ and to limit them. Similarly, to maximize $\tau_{a}$ beyond the MPC horizon, the SoC at $k=48 h$ is forced to be higher than $40 \%$.

$$
-\left|\max \left(P_{\text {bat }}^{M A X}, P_{\text {surplus }}\right)\right| \leq P_{\text {bat }, k}^{c h} \leq 0 ; 0 \leq P_{\text {bat }, k}^{\text {dis }} \leq\left|\max \left(P_{\text {bat }}^{M A X}, P_{\text {deficit }}\right)\right|
$$

\section{Real-time model identification algorithm}

Most of the scientific studies [5] estimate $S o C_{k+1}$ through a model composed of time-invariant parameters derived from batteries' technical specification, as formulated in (5). However, based on more realistic models of Li-ion batteries [13], the efficiency during its charge $\left(\eta_{c h}\right)$ or discharge $\left(\eta_{\text {dis }}\right)$, the 
nominal capacity $\left(Q_{\text {nom }}\right)$ and the nominal voltage $\left(v_{\text {nom }}\right)$ change according to the intensity of current, battery age and cell temperature $\left(T_{\text {cell }}\right)$. According to [7], [9] the voltage variation can be around 10\% of the nominal voltage when they are fully charged and discharged. Moreover, the nominal capacity can reach, at the end of their life, up to $80 \%$ of its initial value [9]. Consequently, additional uncertainties on $S o C_{k+1}$ estimation arise, which may result in under or overuse of the batteries.

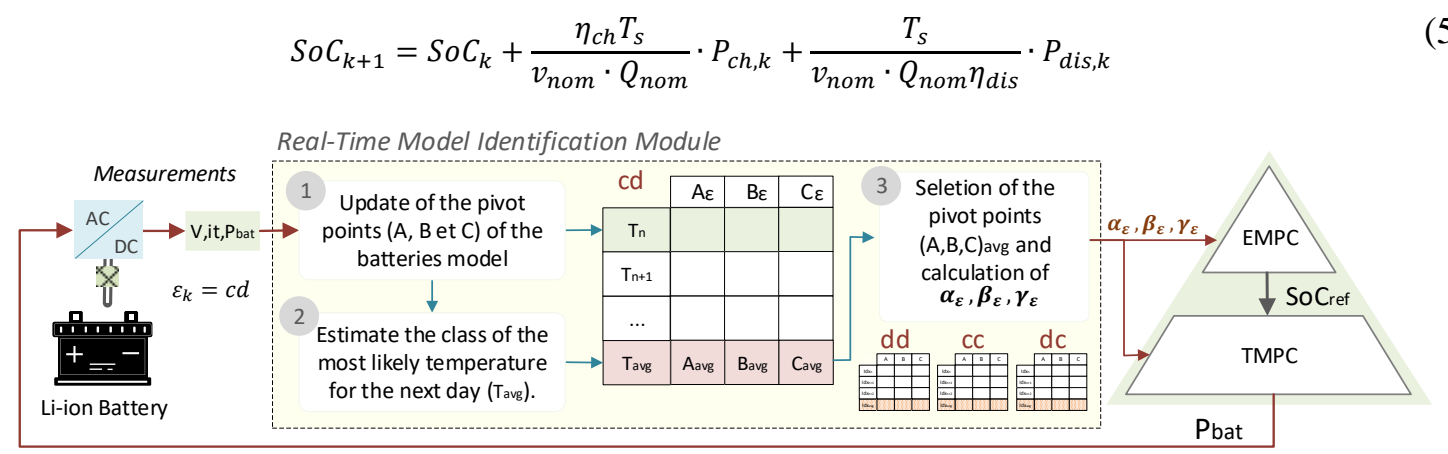

Fig. 3: Real-time parameters identification algorithm for a charge and discharge sequence $\left(\varepsilon_{k}=c d\right)$.

To tackle this issue, the authors designed the algorithm RTMI to improve the estimation of both $S o C_{k+1}$ and their upper $\left(S o C_{\max }^{k+1}\right)$ and lower boundaries $\left(S o C_{\min }^{k+1}\right)$. The main objective of this new algorithm is to reduce human intervention for model calibration and BMG maintenance while taking full advantages of batteries. Therefore, the RTMI module updates the parameters of $S o C_{k+1}$, as illustrated in Fig. 3. The limits $S o C_{\max }^{k+1}$ and $S o C_{\min }^{k+1}$ are determined to guarantee the operation of the batteries in the linear region, as shown in Fig. 4a.

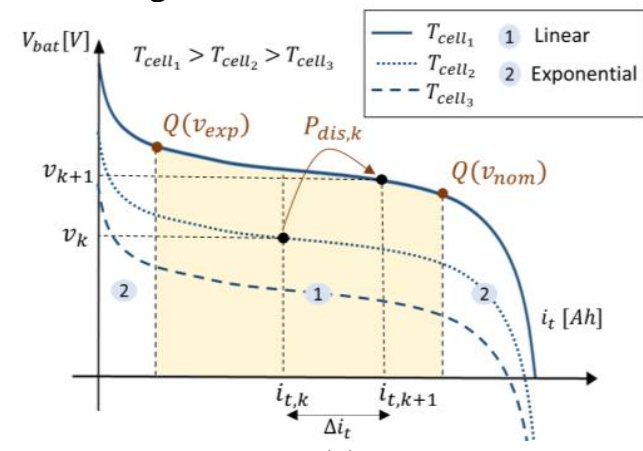

(a)

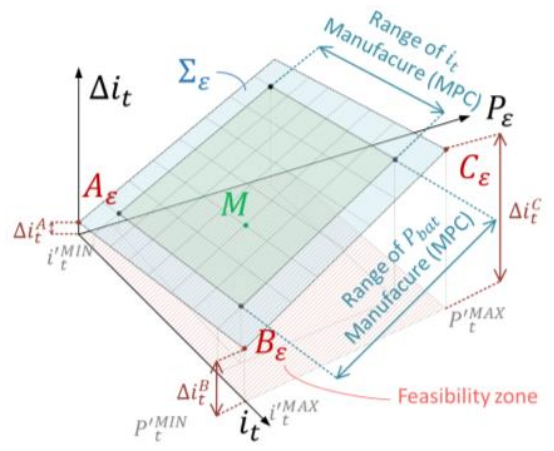

(b)

Fig. 4: RTMI module operation. (a) Discharge curves of a Li-ion battery under temperature cell variations. (b) Model perspective with the three pivot points for predicting the variation of $\operatorname{SoC}\left(\Delta i_{t}\right)$.

The RTMI module determines the parameters $\alpha_{\varepsilon}, \beta_{\varepsilon}$ and $\gamma_{\varepsilon}$ of the $S o C$ model defined by (6) and (7) by implementing an unconventional linear regression based on previous data measurements. The variable $\delta_{\varepsilon, k}$ is a Boolean variable which is worth 1 when the battery is active and 0 otherwise. Remarkably, equation (7) can be interpreted as four surfaces $\Sigma_{\varepsilon}$ of axis $x y z_{\varepsilon}=\left[P_{\varepsilon}, i_{t}, \Delta i_{t}\right]_{\varepsilon}$, as illustrated in Fig. 4b. Then, each surface $x y z$ can be represented by three pivot points, namely $A_{\varepsilon}=\left[i_{t}^{M I N}, P_{b a t}^{M I N}, \Delta i_{t}^{A}\right]_{\varepsilon}$, $B_{\varepsilon}=\left[i_{t}^{M A X}, P_{b a t}^{M I N}, \Delta i_{t}^{B}\right]_{\varepsilon}$ and $C_{\varepsilon}=\left[i_{t}^{M A X}, P_{b a t}^{M A X}, \Delta i_{t}^{C}\right]_{\varepsilon}$, which cover the feasibility zone which corresponds to the limits recommended by the manufacturer extended in $20 \%$. To reduce the impact of voltage variation, the surfaces $\Sigma_{\varepsilon}$ are classified into four main categories, named $\varepsilon=\{c d, d d, c c, d c\}$, which indicate the sequence of batteries' charge $(c)$ and discharge $(d)$, at time $k-1$ and $k$. Consequently, the RTMI sends daily to the HMPC only the values of $\alpha_{\varepsilon}, \beta_{\varepsilon}$ and $\gamma_{\varepsilon}$ corresponding to the most likely temperature for the next day, which is the average temperature of the previous day.

$$
\begin{gathered}
S o C_{k+1}=1-\frac{i_{t, k}+\Delta i_{t, k}}{Q_{n o m_{k}}} ; P_{b a t, k}=P_{b a t, k}^{c h}+P_{b a t, k}^{d i s}=\sum_{\varepsilon=\{c d, d d,, c c, d c\}} P_{\varepsilon, k} \\
\Delta i_{t, k}=\sum_{\varepsilon=\{c d, d d,, c c, d c\}} \alpha_{\varepsilon} P_{\varepsilon, k}+\beta_{\varepsilon} i_{t, k-1}+\gamma_{\varepsilon} \delta_{\varepsilon, k}
\end{gathered}
$$




\section{Details of the RTMI algorithm}

Synchronized with both EMPC and TMPC, the RTMI module acquires a new measurement point $M$, composed by the integral of the current $\left(i_{t}\right)$, the reference power $\left(P_{b a t}\right)$ and the battery voltage $(v)$ at each TMPC sampling time (i.e. $T_{s}^{T M P C}$ ). As detailed in the following sub-sections, the RTMI algorithm is divided into three steps, namely: classification, updating and identification of the limits for charging and discharging the batteries.

\section{Step 1: Classification of data measurements by temperature interval}

As depicted in Fig. $4 \mathrm{a}, v$ is directly correlated to the $T_{\text {cell }}$. This effect in the battery voltage impacts the batteries round-trip efficiency, reducing the accuracy of the classical model defined by (7). Higher voltage when charging the batteries or lower voltages when discharging them implies a loss of efficiency, once the storage energy variation is mainly dependent on the current flowing through the batterie cells. Therefore, aiming to improve the robustness against $T_{\text {cell }}$ disturbance, the RTMI fits a linear model for each temperature ranges using classified measurements points. Without using any temperature sensor, the RTMI algorithm estimates the temperature interval from $v$ measurements. The Fig. 4a and Fig. 5a show that the $T_{\text {cell }}$ mainly involves a vertical offset in the batteries discharge curve, but almost does not affect the slope of $v$ with respect to $i_{t}$. In this way, the maximum amplitude of $v$ $\left(\Delta v_{M A X}\right)$ due to the full charges and discharges of the batteries is almost unchanged, resulting in a quasiconstant slope along with different $T_{\text {cell }}$, as depicted in Fig. 5b and Fig. 5c.

Consequently, when the BMG operates for the first time, the EMS will force two full charges and discharges of the batteries to determine $\Delta v_{M A X}$, and therefore, the absolute value of the slope of the discharge curve $\left(\left|\Delta v / i_{t}\right|\right)$. After this preliminary step, the temperature intervals are defined by identifying the lines $\vec{S}_{T_{\text {class }}}$, as shown in Fig. $5 \mathrm{c}$, where $T_{\text {class }}$ is the identification number for a specific temperature class. The lines $\vec{S}_{T_{\text {class }}}$ are outspread from a voltage interval which is chosen a priori regarding the desired precision, the computing resources and the RTMI convergence time. Typically, this voltage offset can be set as $1 \%$ of $v_{\text {nom }}$. As a result, a measurement $M$ belongs to $T_{\text {class }}$, if and only if, the point $M$ is between the lines $\vec{s}_{T_{\text {class }}}$ and $\vec{s}_{T_{\text {class }}+1}$.

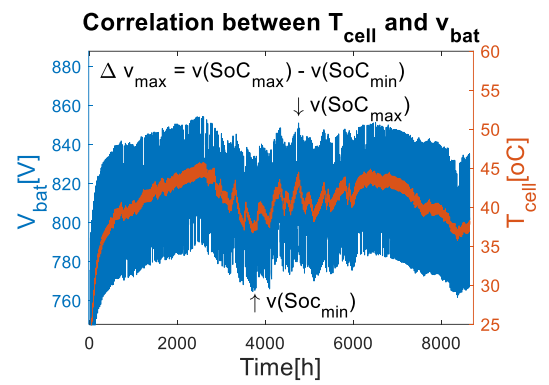

(a)

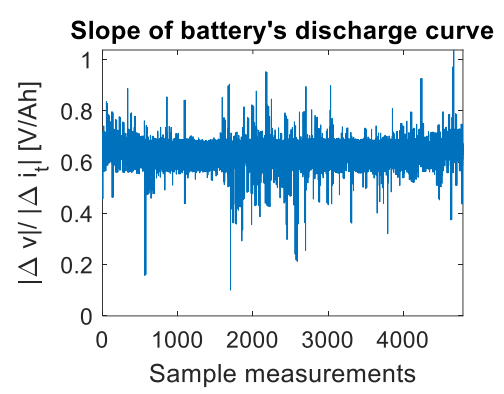

(b)

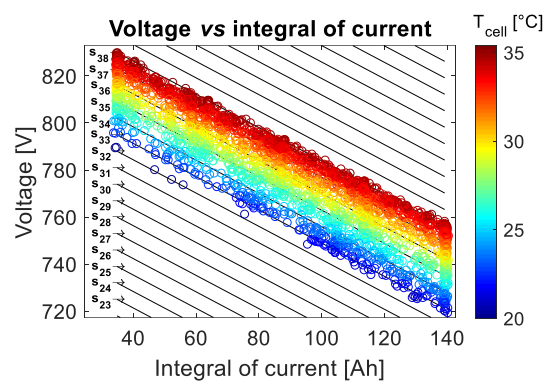

(c)

Fig. 5: One-year simulation of the $\mathrm{BMG}$ with $\mathrm{SoC}_{\max }=80 \%$ and $\mathrm{SoC}_{\min }=20 \%$. (a) $v$ and $T_{\text {cell }}$ correlation. (b) Slope of battery discharge curve. (c) Correlation between_voltage measurements, current integral and $T_{\text {cell }}$.

\section{Step 2: Updating the values of $\alpha_{\varepsilon}, \beta_{\varepsilon}$ and $\gamma_{\varepsilon}$}

Once determined which class of temperature the measurement $M$ belongs to, it is possible to adjust the values of $\alpha_{\varepsilon}, \beta_{\varepsilon}$ and $\gamma_{\varepsilon}$ of these respective $T_{\text {class }}$. The determination of $\alpha_{\varepsilon}, \beta_{\varepsilon}$ and $\gamma_{\varepsilon}$ is implemented based on an iterative and real-time process. As soon as a new measurement $M$ is acquired, the parameters of $\alpha_{\varepsilon}, \beta_{\varepsilon}$ and $\gamma_{\varepsilon}$ are updated. Remarkably, $\alpha_{\varepsilon}, \beta_{\varepsilon}$ and $\gamma_{\varepsilon}$ are vectors of size equals to the number of $T_{\text {class }}$, where each element of these vectors is the fitted values of the model defined by (8) over all measurements $M$. The upmost advantage of the RTMI algorithm is the non-dependency on storage of the past measurements. The knowledge of the previous measures is stored in dynamic confidence weights, named $\omega_{A, k}, \omega_{B, k}$ and $\omega_{C, k}$. 
Starting from minimal values - typically between 0.1 and 1.0 - these confidence weights grow with the acquisition of new measurements. Since the pivot points possess fixed values in the plan $x y=\left[P_{\varepsilon}, i_{t}\right]$, the fitting process only consists of calculating the values of z-axis (i.e. $\Delta i_{t, k}^{A}, \Delta i_{t, k}^{B}$ and $\Delta i_{t, k}^{C}$ ). For the first iteration, the pivot points are initialized according to (8), where it is used the parameters given by the technical specification of the batteries and using the classical model defined in (7).

$$
\Delta i_{t, k=0}^{A}=\Delta i_{t, k=0}^{B}=\frac{\eta_{c h} T_{S}}{Q_{\text {nom }} v_{\text {nom }}} \cdot P_{b a t}^{M I N} ; \Delta i_{t, k=0}^{C}=\frac{T_{S}}{Q_{\text {nom }} v_{\text {nom }} \eta_{\text {dis }}} \cdot P_{b a t}^{M A X}
$$

Thereafter, the values of $\Delta i_{t, k}^{A}, \Delta i_{t, k}^{B}$ and $\Delta i_{t, k}^{C}$ are updated so that to approach the surface $\Sigma_{\varepsilon}$ to the new measurement $M$. To achieve this, an intermediate surface $\phi_{\varepsilon}$ defined by the intermediary pivot points $A_{\varepsilon}^{\prime}, B_{\varepsilon}^{\prime}$ and $C_{\varepsilon}^{\prime}$, with z-axis values equal to $z_{A}, z_{B}$ and $z_{C}$, are calculated through the optimisation of the cost function defined by (9). The surface $\phi_{\varepsilon}$ contains the point $M$ and is determined to make $\Delta i_{t, k+1}^{A, B, C}$ of the next iteration as close as possible of $\Delta i_{t, k}^{A, B, C}$ of the previous period.

$$
z_{A}, \mathrm{z}_{\mathrm{B}}, \mathrm{z}_{\mathrm{C}}=\arg \left(\min _{z_{A,}, \mathrm{z}_{\mathrm{B}}, \mathrm{z}_{\mathrm{C}}} n_{A, k}\left(\Delta i_{t, k}^{A}-z_{A}\right)^{2}+n_{B, k}\left(\Delta i_{t, k}^{B}-\mathrm{z}_{\mathrm{B}}\right)^{2}+n_{C, k}\left(\Delta i_{t, k}^{C}-\mathrm{z}_{\mathrm{C}}\right)^{2}\right)
$$

Subject to:

$$
M \in A_{\varepsilon}^{\prime} B_{\varepsilon}^{\prime} C_{\varepsilon}^{\prime} ; z_{A}, z_{B}, z_{C} \geq 0
$$

Intuitively, high value of $n_{A, k}$ and low values of $n_{B, k}$ and $n_{C, k}$ lead $z_{A}$ approach to $\Delta i_{t, k}^{A}$ faster than $z_{B}$ and $z_{C}$ to $\Delta i_{t, k}^{B}$ and $\Delta i_{t, k}^{C}$, whereas balanced values of $n_{A, k}, n_{B, k}$ and $n_{C, k}$ result in a fair variation among $z_{A}, z_{B}$ and $z_{C}$. The weights of (9) are calculated with regard the normalized distance between the measure $M$ and the respective intermediate pivot point projected on the plan $x y$ (i.e. operator $\|\cdot\|_{x y}$ ), as defined in (10) for the pivot point $A_{\varepsilon}^{\prime}$.

$$
n_{A, k}=\frac{\|\overline{M A}\|_{x y}}{\|\overline{M A}\|_{x y}+\|\overline{M B}\|_{x y}+\|\overline{M C}\|_{x y}}
$$

Once determined the intermediary plan $\phi_{\varepsilon}, \Delta i_{t, k}^{A}, \Delta i_{t, k}^{B}$ and $\Delta i_{t, k}^{C}$ and the confidence weights $\omega_{A, k}, \omega_{B, k}$ and $\omega_{C, k}$ are updated following equations (11) - (14), in the order of compilation. For the sake of simplicity, in this paper, it will only be detailed the updating process of the pivot point $A_{\varepsilon}$, but it is important to highlight that similar equations are used for $B_{\varepsilon}^{\prime}$ and $C_{\varepsilon}^{\prime}$.

$$
\begin{gathered}
\omega_{A, k+1}=\omega_{A, k}+\frac{\frac{1}{\|\overline{M A}\|_{x y}}}{\frac{1}{\|\overline{M A}\|_{x y}}+\frac{1}{\|\overline{M B}\|_{x y}}+\frac{1}{\|\overline{M C}\|_{x y}}} \\
\omega_{\text {inertia, } A}^{A}=\sigma\left(\omega_{A, k+1}-\omega_{A, k}\right) \leq \omega_{A, k+1} \\
\Delta i_{t, k+1}^{A}=\frac{\Delta i_{t, k}^{A} \cdot \omega_{\text {inertia }, k}^{A}+z_{\mathrm{A}} \cdot \omega_{A, k+1}}{\omega_{\text {inertia }, k}^{A}+\omega_{A, k+1}} \\
\omega_{A, k+1}=\omega_{A, k+1}-\tau_{\text {decay }} \cdot\left(\omega_{A, k+1}-\omega_{\text {min }}\right)
\end{gathered}
$$

Firstly, the confidence weight $\omega_{A, k}$ is updated using (11). The closer $M$ is to the $A_{\varepsilon}$, the more reliable the value of $z_{A}$ calculated from (9) is. Consequently, $\omega_{A, k+1}$ is inversely proportional to the distance between the pivot point $A_{\varepsilon}$ and the measurement $M$, and it is normalised according to the distance from other pivot points (i.e. $B_{\varepsilon}$ and $C_{\varepsilon}$ ). To improve the robustness against measurement noise, the updated $\Delta i_{t, k+1}^{A}$ is a weighted value between the previous $\Delta i_{t, k}^{A}$ and the new fitted $z_{A}$. The inertial factor handles the ponderation among these two variables $\omega_{\text {inertia, } k}^{A}$ and the confidence weight $\omega_{A, k+1}$ as defined in (13). The equilibrium amongst $\omega_{\text {inertia, } k}^{A}$ and $\omega_{A, k+1}$ control the convergence time of $\Delta i_{t, k+1}^{A}$ to $\mathrm{z}_{\mathrm{A}}$. This balance can be manually set by tuning the value of $\sigma$, which indicates the importance of the new measurement regarding the previous value. Notably, $\omega_{\text {inertia }, k}^{A}$ is upper limited by $\omega_{A, k+1}$, to restrict the convergence time to a scale of two. Since the charge/discharge curve of batteries changes with age, the confidence weight $\omega_{A, k+1}$ gradually decreases with a time constant $\tau_{\text {decay }}$ until a minimum value $\omega_{\text {min }}$ which is equal to the starting value, as specified in (14). 
After updating the plan $\Sigma_{\varepsilon}$, the coefficients $\alpha_{\varepsilon}, \beta_{\varepsilon}$ and $\gamma_{\varepsilon}$ are calculated using the principles of analytical geometry. As the last step, it is still necessary to refine the developed model, because according to (7) and Fig. $4 \mathrm{~b}$, the plan $\Sigma_{\varepsilon}$ does not cross the $\vec{\imath}_{t}$ axis, which makes batteries model inaccurate for low powers. Therefore, the model polishing consists of forcing the plan $\Sigma_{\varepsilon}$ cross the $\vec{t}_{t}$ axis by using (15).

$$
\Delta i_{t, k}=\sum_{\varepsilon=\{c d, d d, c c, d c\}} \theta_{\varepsilon} P_{\varepsilon, k}=\sum_{\varepsilon=\{c d, d d, c c, d c\}} P_{\varepsilon, k}\left(\alpha_{\varepsilon}+\frac{1}{P_{b a t}^{\prime M A X}} \cdot\left(\gamma_{\varepsilon}+\frac{\left(i_{t}^{\prime M A X}+i_{t}^{\prime M I N}\right) \cdot \beta_{\varepsilon}}{2}\right)\right)
$$

\section{Step 3: Identification of the limits for charging and discharging the batteries}

As mentioned before and illustrated in Fig. 6a, the batteries must operate between points $Q\left(v_{\text {exp }}\right)$ and $Q\left(v_{n o m}\right)$. The strategy consists in adjusting $i_{t, \max }$ and $i_{t, \min }$ to reduce the variations of the slope $\left|\Delta v / \Delta i_{t}\right|$. According to Fig. 6a, while $i_{t}$ is inside the linear zone (zone 1), the slope $\left|\Delta v / \Delta i_{t}\right|$ is quasiconstant, because $v$ is linearly dependent on $i_{t}$. However, when operating outside this zone (zone 2), $\left|\Delta v / \Delta i_{t}\right|$ is not constant because $v$ is non-linear regarding $i_{t}$.

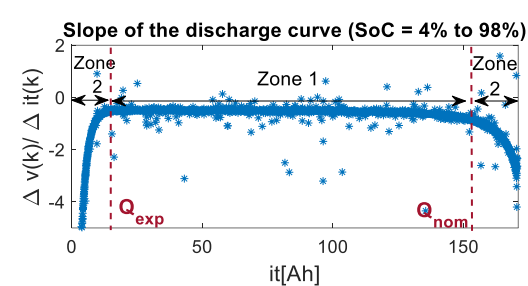

(a)

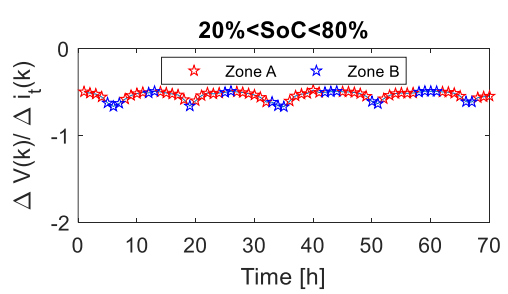

(b)

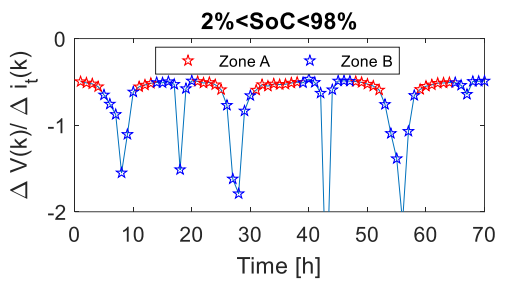

(c)

Fig. 6: Simulation of Li-ion batteries. (a) The slope of the charge/discharge curves as a function of $i_{t}$. (b) and (c) Temporal evolution of the slope when operating in linear and non-linear zones, respectively.

Based on this phenomenon, the developed algorithm for identifying the actual boundaries of $i_{t}$ divides the temporal graphs into two zones, named zone $A$ and zone $B$, as illustrated in Fig. $6 \mathrm{~b}$ and Fig. $6 \mathrm{c}$. The zone $A$, indicated by the red dots, represents the range where the batteries certainly operates in the linear zone. On the other hand, the zone $B$, outlined by the blue dots, is the zone for which the battery can be either in the linear or non-linear region. Therefore, zone A comprehends the range of $20 \%$ to $80 \%$ of the predefined limits of $i_{t, \min }$ and $i_{t, \max }$, whereas zone $B$ is its complementary region.

Remarkably, the oscillation of the slope is more intense when zone B is in the non-linear zone (Fig. 6c) than when it is inside the linear range (Fig. 6b). As a result, to determine whether zone B corresponds to the linear or non-linear range, the average of the absolute difference between each $\left|\Delta v / \Delta i_{t}\right|$ measured inside zone $B\left(m_{u p}\right)$ and those measured inside zone $A\left(m_{\text {down }}\right)$ are constant compared, through the mean deviations $\vartheta_{u p}$ and $\vartheta_{\text {down }}$ calculated as detailed in Fig. 7. These deviations $\vartheta_{u p}$ and $\vartheta_{\text {down }}$ are monitored by means of a proportional controller with a hysteresis at its input to maintain them within the range $\vartheta_{u p \text {, down }}^{\text {ref }} \pm$ Tol. The values of $T o l \cong 2 \%$ and $\vartheta_{u p \text {, down }}^{\text {ref }} \cong 10 \%$ were manually regulated, but they can be re-adjusted to reduce the oscillations or increase the response time of $i_{t, \min }$ and $i_{t, \max }$. If the $i_{t}$ boundaries are modified more than $10 \%$ of the previous values, the feasibility zone of Fig. $4 \mathrm{~b}$ is readjusted accordingly and the confidence values $\omega_{A}, \omega_{B}, \omega_{C}$ are reset to $\omega_{\min }$.

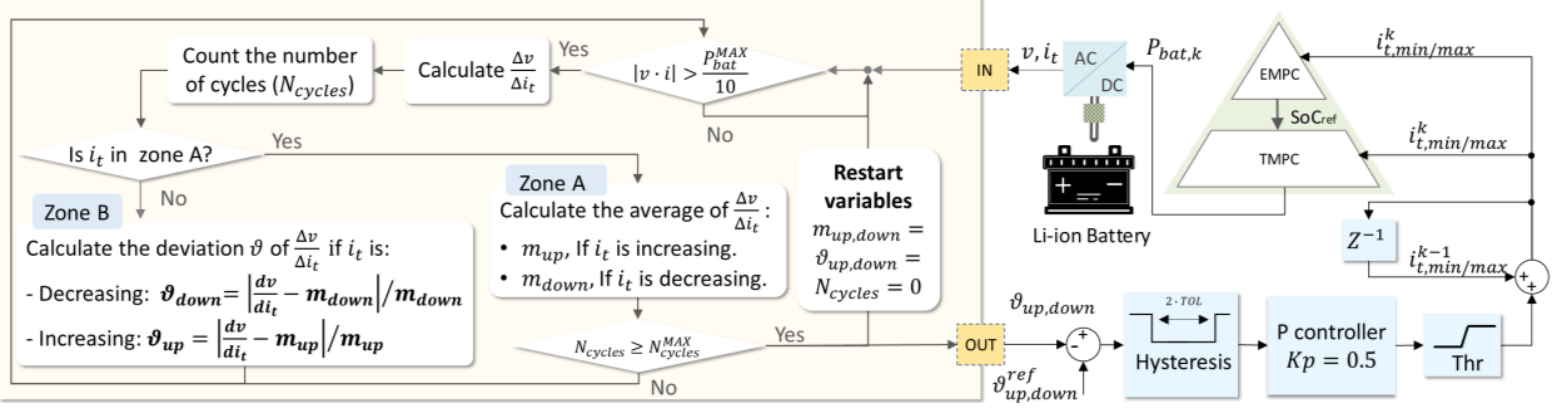

Fig. 7: Algorithm for identifying the $i_{t}$ limits to guarantee the batteries' operation inside the linear zone. 


\section{Simulation results}

Aiming to evaluate the performance of the proposed control architecture, a BMG equipped with PV arrays with $107 \mathrm{kWc}$ and $\mathrm{Li}$-ion batteries with nominal voltage of $700 \mathrm{~V}$ and nominal capacity of $167 \mathrm{Ah}$ was simulated for 365 days in MATLAB Simulink ${ }^{\circledR}$ under several scenarios. The simulations were carried out using real solar radiation data [14] and the estimated annual building energy consumption, resulting in $135.95 \mathrm{MWh}$ energy generation and $241.85 \mathrm{MWh}$ energy consumption per year. The $\tau_{a}$ and the total power exchanged with the main grid was used for assessing the proposed HMPC with RTMI module, a non-hierarchical MPC, and a conventional rule-based (RB) strategy with and without data prediction inaccuracy.

The RB was adapted from [15], where, using no data prediction, the batteries are charged when there is an energy surplus and discharged when there is an energy deficit. The simulation results are divided into two subsections. The first one is to validate the RTMI algorithm for SoC and batteries' capacity estimation, while the second one is for assessing the benefits of dividing the EMS hierarchically.

\section{Performance of the algorithm for batteries' parameter identification}

To show the robustness of the RTMI algorithm against inaccuracies of the parameters coming from the technical specifications, three initial values of $Q$ were considered, namely $Q_{80}, Q_{100}$ and $Q_{120}$, corresponding to $80 \%, 100 \%$ and $120 \%$ of the actual capacity (167 Ah), respectively. To verify the error between the day-ahead $S o C_{r e f}$ calculated by the EMPC and the real one, the TMPC in these scenarios was considered as a perfect router. Consequently, instead of optimising (3), it does implement the control variables determined by EMPC. In this manner, it is possible to decouple the effect of TMPC and highlight only the impact of the errors in EMPC state of charge estimation on the BMG performance. The graph in Fig. 8a shows that, in all study cases, the cumulative error in predicting $S_{o} C_{k+1}$ using the RTMI module is about 3 times lower than using the conventional model with static parameters. Furthermore, the graphs in Fig. 8b shows that the EMPC empowered by RTMI module assures $\tau_{a}$ about $3 \%$ higher than the EMPC without RTMI module with $Q_{80}$ and between $2 \%$ and $4 \%$ higher than RB controller.

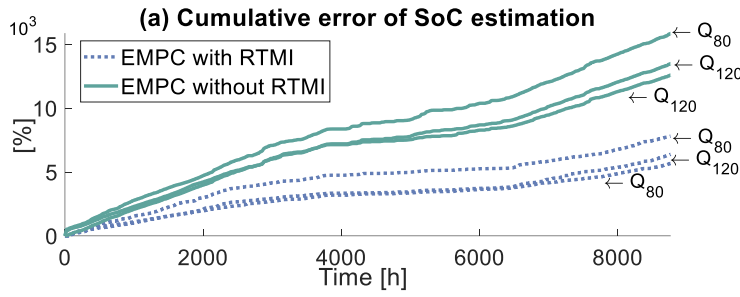

(d) Boundaries of $i_{t}$ and the DoD

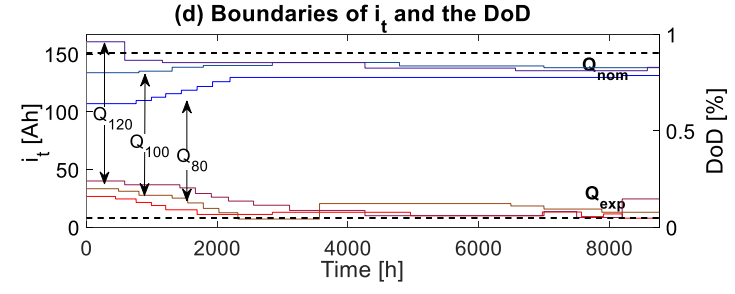

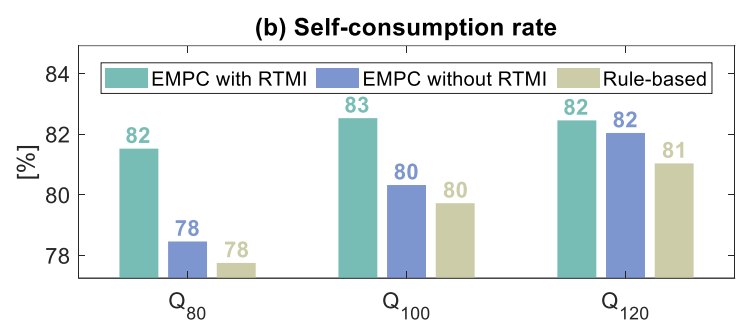

(d) Temperature classes vs cell temperature

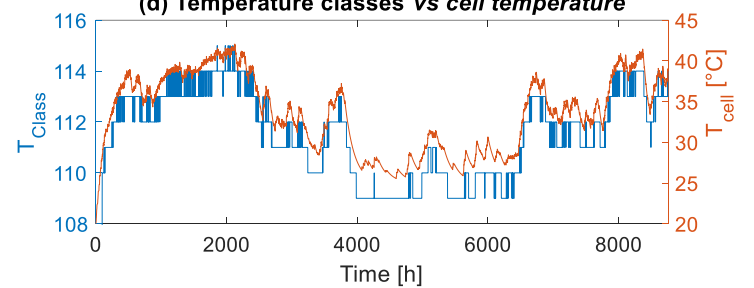

Fig. 8: Evaluation of the EMPC robustness against parameter imprecision. (a) The cumulative error of the SoC estimation. (b) Self-consumption rate comparison. (c) Maximum (in blue lines) and minimum (in red lines) boundaries of the $i_{t}$ when using RTMI. (d) Estimation of $T_{\text {cell }}$ variation through $T_{\text {class }}$.

The increase in $\tau_{a}$ is mainly due to the enlargement of the $i_{t}$ boundaries closed to the real frontiers $\left(Q_{\text {exp }}\right.$ and $Q_{\text {nom }}$ ), as shown in Fig. 8c. Accurately estimating the battery capacity reinforces the potential of batteries of shifting the load toward the periods of energy surplus, resulting in an enhanced internal load matching and lower grid dependency. For this reason, the relative difference between $\tau_{a}$ is more remarkable when the batteries' capacity is underestimated (i.e. $Q_{80}$ ). Nonetheless, there is a tradeoff between fostering $\tau_{a}$ and the batteries' state of heathy. Fig. $8 \mathrm{c}$ shows that expanding $i_{t}$ boundaries, the attained depth of discharge (DoD) is higher when employing RTMI module than not using it (at the 
beginning of the simulation), accelerating the batteries' degradation [7]. In addition, as shown in Fig. $8 \mathrm{~d}$ employing the RTMI module and processing of $v$ and $i_{t}$ measurements, $T_{\text {cell }}$ can be supervised without needing any thermal sensor, because $T_{\text {class }}$ is an image of the temperature variation. This can provide further information to the HMPC for preserving the batteries state of healthy [2], [8].

\section{Performance of the proposed two-level hierarchical control structure}

Since the purpose of the hierarchical EMS is to soften the drawbacks provoked by stochastic variations in the internal power imbalance, the prediction data were multiplied by a random time-dependent factor $(\rho)$, as detailed in Fig. 9a. In this manner, the error in the estimate power imbalance grows according to the horizon, attaining up to $60 \%$ at $N_{h}^{E M P C}=48$, as shown in Fig. $9 \mathrm{~b}$ and Fig. $9 \mathrm{c}$. Aiming to assess the proposed HMPC, five control architectures were investigated. The first and second ones are the HMPC with and without RTMI modules. The third and fourth control structures are the non-hierarchical MPC with and without RTMI module, in which only EMPC is updated hourly with the full horizon. Finally, the fourth control disposal is the simple RB.

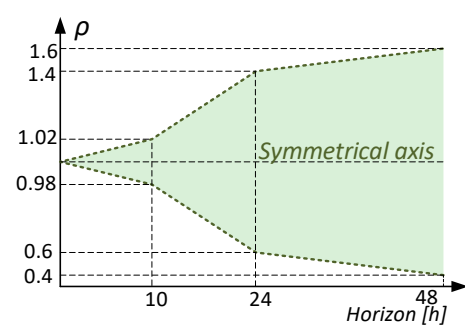

(a)

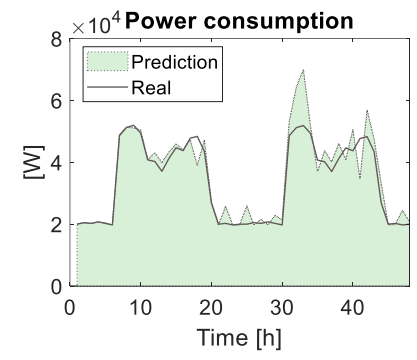

(b)

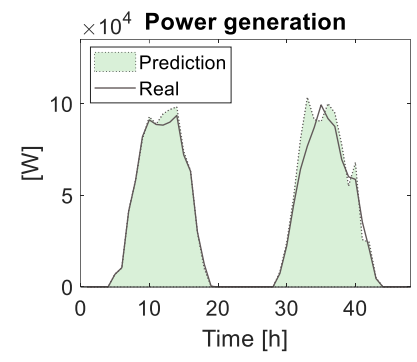

(c)

Fig. 9: Real power and its 48-hours data prediction of a summer day. (a) Time-variant factor. (b) Power consumption. (c) Power generation.

Fig. 10a and Fig. 10b show that both HMPC and the non-hierarchical MPC are robust against data prediction uncertainties, because with and without errors, the BMG imported and exported almost the same amount of energy in a year. Remarkably, when using HMPC with RTMI, it imported about 1\% less energy and exported $8 \%$ less than without RTMI. Even though the non-hierarchical MPC optimises the cost function (2) around 11 times more than the HMPC, the results are very similar. Indeed, the nonhierarchical structure triggers EMPC at each hour, which means 8760 optimizations in a year, whereas only up to 631 times (number of re-optimisations plus once per day) using the proposed control structure, as detailed in Fig. 10c.
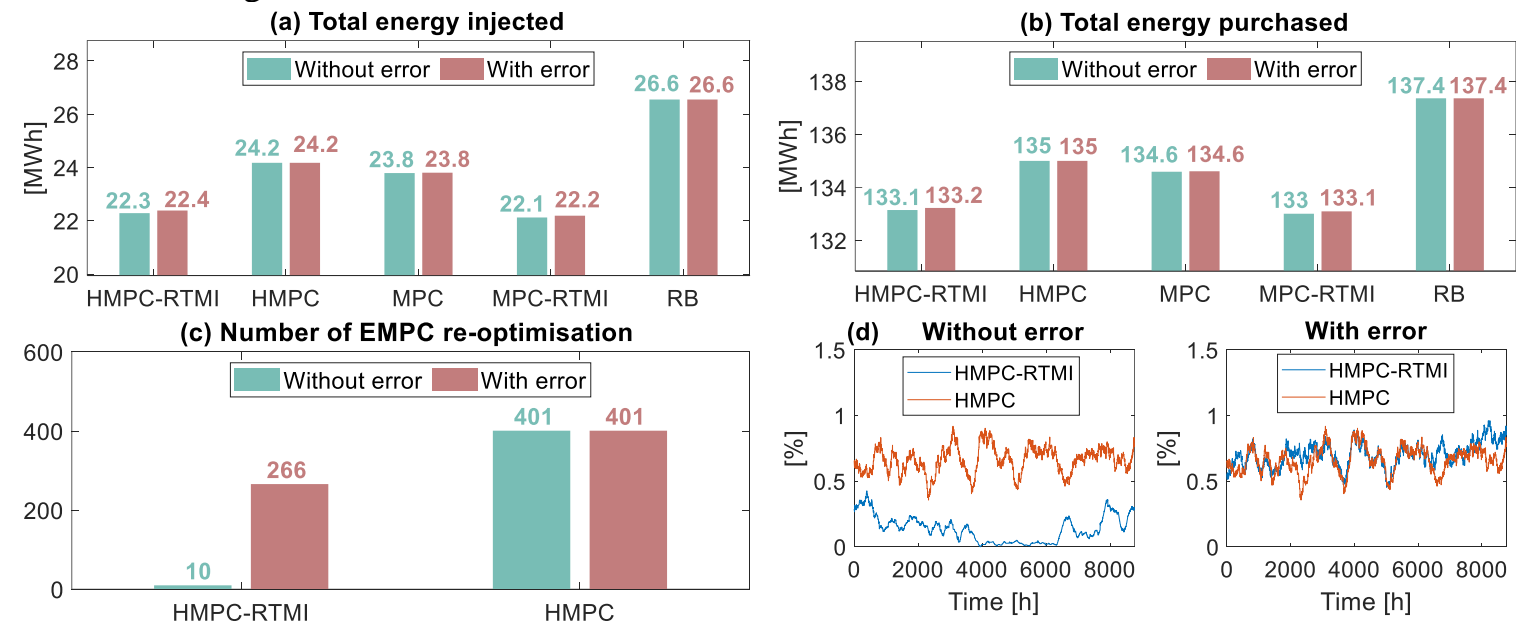

Fig. 10. Comparison between different control architectures. (a) Total power injected. (b) Total power purchased. (c) Number of EMPC re-optimization. (d) Moving average error in $P_{\text {grid }}^{r e f}$ of the last 10 days

The cooperation of two control layers - one with long and another with a short horizon - enables to handle prediction data variability without needing to optimize the laborious cost function (2) every hour, 
but only when the error in either $S o C_{r e f}$ or $P_{\text {grid }}^{r e f}$ is greater than $\Delta_{S o C}^{t h r}=\Delta_{P \text { grid }}^{\text {thr }}=7 \%$. Fig. $10 \mathrm{~d}$ shows that errors in $P_{\text {grid }}^{\text {ref }}$ are higher when not using the RTMI module, because of imprecisions in SoC estimation, which result in 391 more re-optimisations in the scenario without errors and 135 with errors.

\section{Conclusions}

This paper aims to develop a generic Building MicroGrid Energy Management System capable of adapting to external changes, such as Li-ion batteries modelling inaccuracy and inherent power imbalance uncertainties. The hierarchical MPC empowered with the proposed real-time parameter identification module increases the self-consumption rate regarding a well-established rule-based controller and the conventional MPC. This data-driven algorithm enables to identify the original batteries capacity and the cell temperature without any previous modelling step and without any thermal sensor, which simplify the energy management system design. Moreover, the simulation results demonstrated that the division in two control layers reduces the number of optimizations while maintaining the building less energetic dependent on the external grid even under imprecisions in data predictions.

\section{References}

[1] T. M. Lawrence et al., "Ten questions concerning integrating smart buildings into the smart grid," Build. Environ., vol. 108, pp. 273-283, Nov. 2016, doi: 10.1016/j.buildenv.2016.08.022.

[2] G. Cardoso, T. Brouhard, N. DeForest, D. Wang, M. Heleno, and L. Kotzur, "Battery aging in multi-energy microgrid design using mixed integer linear programming," Appl. Energy, vol. 231, pp. 1059-1069, Dec. 2018, doi: 10.1016/j.apenergy.2018.09.185.

[3] M. F. Zia, E. Elbouchikhi, and M. Benbouzid, "Microgrids energy management systems: A critical review on methods, solutions, and prospects," Appl. Energy, vol. 222, pp. 1033-1055, Jul. 2018, doi: 10.1016/j.apenergy.2018.04.103.

[4] Z. Cheng, J. Duan, and M.-Y. Chow, "To Centralize or to Distribute: That Is the Question: A Comparison of Advanced Microgrid Management Systems,” IEEE Ind. Electron. Mag., vol. 12, no. 1, pp. 6-24, Mar. 2018, doi: 10.1109/MIE.2018.2789926.

[5] P. R. C. Mendes, L. V. Isorna, C. Bordons, and J. E. Normey-Rico, "Energy management of an experimental microgrid coupled to a V2G system," J. Power Sources, vol. 327, pp. 702-713, Sep. 2016, doi: 10.1016/j.jpowsour.2016.07.076.

[6] L. Su et al., "Path dependence of lithium ion cells aging under storage conditions," J. Power Sources, vol. 315, pp. 35-46, May 2016, doi: 10.1016/j.jpowsour.2016.03.043.

[7] X. Li, C. Yuan, X. Li, and Z. Wang, "State of health estimation for Li-Ion battery using incremental capacity 10.1016/j.energy.2019.116467.

[8] Z. Song et al., "The sequential algorithm for combined state of charge and state of health estimation of lithium-ion battery based on active current injection," Energy, vol. 193, p. 116732, Feb. 2020, doi: 10.1016/j.energy.2019.116732.

[9] Y. Li et al., "Data-driven health estimation and lifetime prediction of lithium-ion batteries: A review," Renew. Sustain. Energy Rev., vol. 113, p. 109254, Oct. 2019, doi: 10.1016/j.rser.2019.109254.

[10] R. Luthander, J. Widén, D. Nilsson, and J. Palm, "Photovoltaic self-consumption in buildings: A review," Appl. Energy, vol. 142, pp. 80-94, Mar. 2015, doi: 10.1016/j.apenergy.2014.12.028.

[11] Enedis l'électricité en réseau, "Conditions de raccordement des Installations de stockage." [Online]. Available: https://www.enedis.fr/sites/default/files/Enedis-PRO-RES_78E.pdf.

[12] Commission de Régulation de l'Energie, "Cahier des charges de l'appel d'offres portant sur la réalisation et l'exploitation d'Installations de production d'électricité à partir d'énergies renouvelables en autoconsommation et situées en métropole continentale.” Dec. 26, 2019.

[13] O. Tremblay and L.-A. Dessaint, "Experimental Validation of a Battery Dynamic Model for EV Applications," World Electr. Veh. J., vol. 3, no. 2, pp. 289-298, Jun. 2009, doi: 10.3390/wevj3020289.

[14] "JRC Photovoltaic Geographical Information System (PVGIS) - European Commission." https://re.jrc.ec.europa.eu/pvg_tools/en/tools.html\#MR (accessed Mar. 05, 2020).

[15] H. Yu, S. Niu, Y. Zhang, and L. Jian, "An integrated and reconfigurable hybrid AC/DC microgrid architecture with autonomous power flow control for nearly/net zero energy buildings," Appl. Energy, vol. 263, p. 114610, Apr. 2020, doi: 10.1016/j.apenergy.2020.114610. 